\title{
Pyogenic Liver Abscess Following an Uncomplicated Colonoscopy
}

\author{
George Rahmani, Anne-Marie Moran \\ Department of Nephrology and General Medicine, Letterkenny General Hospital, Letterkenny, Ireland
}

How to cite this article: Rahmani G, Moran AM. Pyogenic liver abscess following an uncomplicated colonscopy. EJCRIM 2017;4: doi:10.12890/2017_000565

Conflicts of Interests: The Authors declare that there are no competing interests.

This article is licensed under a Commons Attribution Non-Commercial 4.0 License

\section{ABSTRACT}

Pyogenic liver abscess is a potentially devastating clinical entity associated with significant morbidity and mortality ${ }^{[1]}$. A myriad of causes for liver abscess have been described including intra-abdominal infections such as diverticulitis ${ }^{[2]}$. Due to a non-specific presentation, clinicians often require a high level of suspicion in their diagnosis of this condition. A handful of cases of liver abscess have been described following colonoscopy which was usually a complicated procedure or one where multiple biopsies had been taken ${ }^{[3,4]}$. The case of a patient presenting pyrexia of unknown origin one week after undergoing an uncomplicated colonoscopy in which no biopsies were taken is reported. She was ultimately diagnosed with a pyogenic liver abscess.

\section{LEARNING POINTS}

- Pyogenic liver abscess is an important differential when investigating pyrexia of unknown origin.

- Liver abscesses can rarely occur following colonoscopy.

\section{KEYWORDS}

Liver abscess, colonoscopy, MRI, ultrasound

\section{CASE PRESENTATION}

A 67-year-old previously healthy female was admitted with a two-day history of rigors, nausea, vomiting, and generalized abdominal pain. She had a colonoscopy to investigate diarrhea one week prior to the onset of her symptoms. The colonoscopy was reported as being essentially normal. Stool cultures at the time were negative for any clinically relevant pathogenic organism such as Campylobacter, Salmonella, Shigella species, Clostridium difficile, or the particularly pathogenic strain O157:H7 of Escherichia coli. There was no history of recent foreign travel. The patient appeared unwell on admission. She was pyrexial $\left(38.7^{\circ} \mathrm{C}\right)$ and tachycardic $(109 \mathrm{BPM})$. She complained of generalized abdominal pain. Physical examination was otherwise unremarkable. Her white cell count was elevated (13.13 x10\%/L) as was her C-reactive Protein $(67 \mathrm{mg} / \mathrm{L})$. Her liver and renal profiles were within the normal physiological ranges. Urine dipstick was negative for blood, leucocytes, and nitrites. She deteriorated on day two with swinging fevers and rigors (chills). Neither blood cultures nor urine cultures yielded any positive results, and intravenous piperacillin/tazobactam and gentamicin was commenced and eventually changed to IV meropenem and vancomycin, a regimen which the patient seemed to respond well to.

Her abdominal pain persisted and computed tomography (CT) scan of the abdomen and pelvis was performed in an attempt to identify a source of infection. The CT scan demonstrated several cystic lesions in the liver (Fig. 1). Of note, the patient had a history of liver cysts, which had been picked up incidentally on an ultrasound of the biliary system several years previously (Fig. 2). 
An ultrasound of the liver and magnetic resonance cholangiopancreatography (MRCP) were carried out to further investigate these lesions in greater detail. In fact, the MRCP revealed that one of the larger cysts had most likely become a pyogenic liver abscess (Fig. 3). This was promptly drained under ultrasound guidance and the patient was given a further two weeks of IV meropenem and vancomycin postdrainage. Given that the patient had received almost a week of IV antibiotics before drainage, the pus was sterile when sent for culture. The patient improved significantly following drainage and was subsequently discharged.

The authors postulate that the well-described phenomenon of transient bacteremia following colonoscopy ${ }^{[5,6]}$ may have seeded infection in one of the patient's pre-existing hepatic cysts, thereby converting a simple benign cyst into a pyogenic liver abscess.

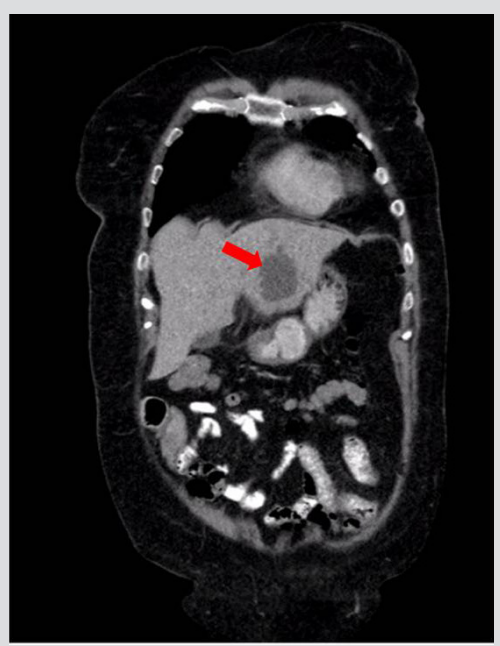

Figure 1. CT Scan Coronal Section - 5cm cyst in the left lobe of the liver (red arrow)

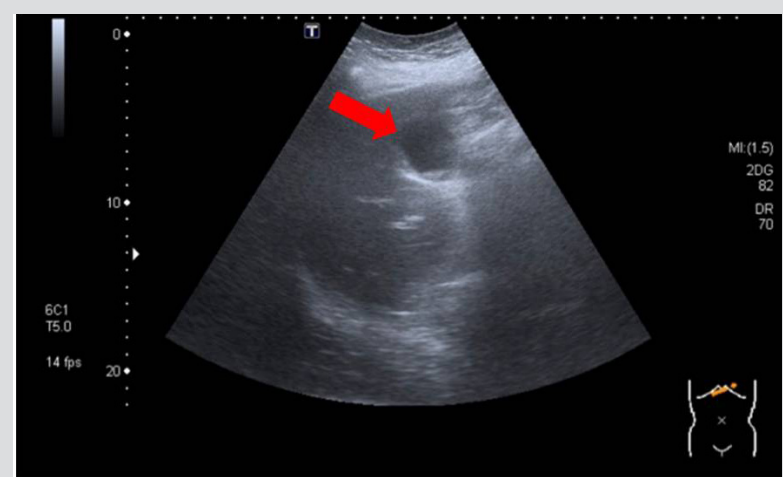

Figure 2. Ultrasound Scan - 5cm loculated cyst in the left lobe of the liver (red arrow)

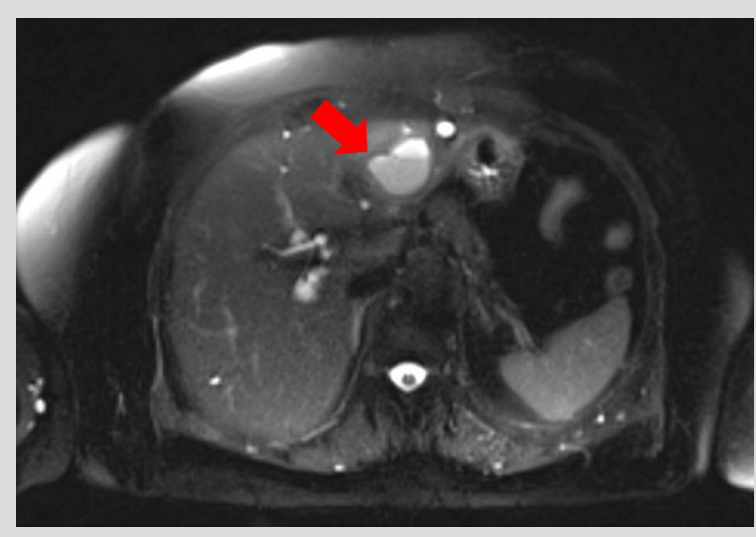

Figure 3. MRCP Axial Section (T2 HASTE Sequence) - $5 \mathrm{~cm}$ lobulated liver abscess in the lateral segment of the left lobe of the liver (red arrow).

\section{DISCUSSION}

The incidence of pyogenic liver abscess (PLA) is in the region of 10 to 20 per $100,000^{[1]}$. Infection can spread to the liver by multiple routes including from the biliary tree, the hepatic artery, portal venous circulation, or direct spread from a nearby focus of infection or penetrating trauma. The route of invasion may also originate from the bowel, either by local extension or via hematogenous seeding. An early diagnosis of PLA is important for prognosis as it has a mortality rate of approximately $10 \%{ }^{[7]}$. Given its largely nonspecific presentation, a diagnosis of PLA can prove challenging unless the clinician maintains a high level of suspicion for this condition. Patients may present with pyrexia, right upper quadrant pain, and/or signs of peritonism, but these signs are neither sensitive nor specific ${ }^{[8]}$.

Infectious complications following colonoscopy are rare. However, several cases have been reported in the literature. These reports include PLA occurring as a complication of sigmoid polypectomy ${ }^{[9]}$ and also following the removal of an impacted fish bone ${ }^{[3]}$. To the best knowledge of the authors, this is the first report of a sterile liver abscess following an uncomplicated colonoscopy. 


\section{REFERENCES}

1. Johannsen EC, Sifri CD, Madoff LC. Pyogenic liver abscesses. Infect Dis Clin North Am 2000;14:547-63, vii.

2. Murarka S, Pranav F, Dandavate V. Pyogenic liver abscess secondary to disseminated streptococcus anginosus from sigmoid diverticulitis. J Glob Infect Dis 2011:3:79-81.

3. Paraskeva KD, Bury RW, Isaacs P. Streptococcus milleri liver abscesses: an unusual complication after colonoscopic removal of an impacted fish bone. Gastrointest Endosc 2000;51:357-8.

4. Ross JJ, Cooley JS, Lee DL. Septic thrombophlebitis of the hepatic veins after colonoscopy: a case report. Gastrointest Endosc 2003;581:155-6.

5. Low DE, Shoenut JP, Kennedy JK, Sharma G P, Harding GK et al. Prospective assessment of risk of bacteremia with colonoscopy and polypectomy. Dig Dis Sci 1987;32:1239-43.

6. Nelson DB. Infectious disease complications of GI endoscopy: part II, exogenous infections. Gastrointest Endosc 2003;57:695-711.

7. Meddings L, Myers RP, Hubbard J, Shaheen AA, Laupland KB, et al. A population-based study of pyogenic liver abscesses in the United States: incidence, mortality, and temporal trends. Am J Gastroenterol 2010;105: 117-24.

8. Pang TC, Fung T, Samra J, Hugh TJ, Smith RC. Pyogenic liver abscess: an audit of 10 years' experience. World J Gastroenterol 2011;17:1622-30.

9. Lai HC, Chan CY, Peng CY, Chen, CB, Huang WH. Pyogenic liver abscess associated with large colonic tubulovillous adenoma. World J Gastroenterol 2006;12:990-2. 